\title{
Electron temperature probe onboard Japan's Mars orbiter
}

\author{
Koh-ichiro Oyama ${ }^{1}$, Takumi Abe ${ }^{1}$, Kristian $\mathrm{Schlegel}^{2}$, Andrew Nagy ${ }^{3}, \mathrm{Jhoon} \mathrm{Kim}^{4}$, and Katsuhide Marubashi ${ }^{5}$ \\ ${ }^{1}$ Institute of Space and Astronautical Science, 3-1-1 Yoshinodai, Sagamihara, Kanagawa 229-8510, Japan \\ ${ }^{2}$ Max Planck Institute for Aeronomy, Katlenburg, Lindau, Germany \\ ${ }^{3}$ Atmospheric and Oceanic Environment Laboratory, Univ. of Michigan, Ann Arbor, Michigan, U.S.A. \\ ${ }^{4}$ Korean Aerospace Research Institute, 52 Eoeundong, Yusung-ku, Taejun, 305-333, Korea \\ ${ }^{5}$ Communication Research Laboratory, 4-2-1 Nukui-kitamachi, Koganei 184-8795, Japan
}

(Received August 7, 1997; Revised August 28, 1999; Accepted October 6, 1999)

\begin{abstract}
Japan' s first Mars spacecraft PLANET-B was successfully launched on 4th of July, 1998 and was named "NOZOMI" after the launch. One of the scientific instruments is a unique electron temperature probe which was developed in Japan and has been used for more than 20 years on sounding rockets as well as on scientific satellites (Oyama, 1991). The electron temperature probe dubbed PET (Probe for Electron Temperature measurements) consists of two planar electrodes, $150 \mathrm{~mm}$ in diameter, placed at the edges of the two solar cell panels of the "NOZOMI" spacecraft. Electron temperatures can be measured in plasmas with densities exceeding $1000 \mathrm{~cm}^{-3}$ with sufficient accuracy. The maximum sampling rate of 8 data points per satellite spin for each probe allows high resolution measurements (i.e., an angular resolution around the spin axis of 23 degrees). Additionally, the probe can measure the anisotropy of the electron temperature, if it exists. It is also possible to infer the existence of nonthermal electrons.
\end{abstract}

\section{Scientific Objectives}

Japan's first Mars orbiter was successfully launched on 4th of July, 1998 and was called "NOZOMI" after the launch. Although the "NOZOMI" was supposed to be placed into a Mars orbit in November 1999, the arrival at the Mars was now rescheduled to 2004 due to the malfunction of the RCS (Reaction Control System). The highly elliptical NOZOMI orbit with periapsis altitude of $150 \mathrm{~km}$ and apoapsis distance of 15 Martian radii makes it possible to study the interaction between the solar wind and the Martian atmosphere by means of 14 different instruments. The orbital inclination of 170 degrees with respect to the ecliptic plane is chosen so that the subsolar ionosphere can be sampled during at least one of the NOZOMI periapsis paths during the design life and also to keep the solar eclipse time within the allowed period limit. One of the scientific instruments carried onboard is a device to measure electron temperatures in the Martian ionosphere, and it is called PET (Probe for Electron Temperature measurements).

To date only two height profiles of electron temperatures of the Mars ionosphere exist; they were derived from the electron energy spectra measured with a retarding potential analyzer onboard the Viking landers (Hanson and Mantas, 1988). This extremely limited data base indicates that we know very little about the energetics of the Martian ionosphere. Therefore we believe that the successful measurement of electron temperature variations in the ionosphere of Mars using PET will significantly contribute to our understanding not only of the energetics, but also of the dynamics

Copy right (C) The Society of Geomagnetism and Earth, Planetary and Space Sciences (SGEPSS); The Seismological Society of Japan; The Volcanological Society of Japan; The Geodetic Society of Japan; The Japanese Society for Planetary Sciences. of the Mars plasma environment.

One of the important issues of the Mars environment concerns the nature and/or existence of an ionopause. Dayside electron density profiles obtained in situ with the Pioneer Venus Orbiter, as well as radio occultation methods starting with Mariner 5, usually show a distinct ionopause on Venus. On the other hand, the radio occultation measurements at Mars obtained with Viking 1 and 2 and Mariners 4, 6, 7 and 9 have never revealed a credible pressure balance at the ionopause (Zhang et al., 1990a,b). Thus there is apparently a real difference in the solar wind interaction processes at the two planets. Hanson and Mantas (1988) concluded from the Viking Lander data that the sum of all the measured partial plasma pressures fails to balance the measured electron pressure near $1000 \mathrm{~km}$ altitude by a factor of about 4 . If the Martian ionopause is located at high altitudes $(\geq 1000 \mathrm{~km})$, the electron temperature should be around $12,000 \mathrm{~K}$ in order to balance the solar wind without an intrinsic magnetic field. Electron temperatures obtained by the PET instrument and electron densities obtained from plasma wave measurements can help to elucidate the still outstanding question concerning the plasma pressure issue.

Hanson and Mantas (1988) separated three populations of electrons with different temperatures using a sophisticated analysis of the data obtained with the retarding potential analyzer on Viking 2. The PET probe can provide an indication of the existence of a high energy tail in the electron energy distribution or deviation from a Maxwellian distribution, if it exists.

The third research topic concerns the existence of "holes" in the Mars ionosphere, similar to the ones found in the Venus ionosphere. These holes may not be present in the Mars ionosphere, if the fluctuating magnetic field carried by the 
solar wind does not penetrate into the ionosphere because of a weak intrinsic magnetic field of Mars. In a recent paper, Cole (1994) argued that flux ropes are not produced at the ionopause of Venus and convected to their place of observation, thus providing evidence against the suggestion that they are caused by the Kelvin-Helmholtz instability at the ionopause. The formation mechanism of the holes in the Venus ionosphere, where the energy densities of the magnetic field and plasma are believed to be in balance, is therefore still controversial. Electron temperature observations by our probe, both inside and outside of the holes (if they exists on Mars) surely provide a chance for a better understanding of this problem.

Theoretical calculations have shown that in order to explain the observed daytime electron temperatures on Venus and Mars, either the heat conduction has to be decreased and/or an extra energy source, beyond EUV heating, needs to be assumed (Choi et al., 1998). The use of the expression for thermal conductivity, appropriate for a fully ionized gas, appears to be inappropriate for model calculations. The fluctuation of the magnetic field in the Mars ionosphere and/or the presence of a horizontal magnetic field can reduce the heat conduction (Hoegy et al., 1990; Cravens et al., 1980). The assumption of such a modified thermal conductivity can be studied by means of d.c. and fluctuating magnetic field data which will be provided by the magnetometer and the wave receiver onboard "NOZOMI". Measurements of electron temperature of the nighttime Martian ionosphere itself, in spite of the limited height range, might be useful in order to find some clues to the cause of elevated electron temperature of the nighttime ionosphere of Venus. The Pioneer Venus orbiter results show that both nighttime electron and ion temperatures are equal to or higher than the daytime temperatures.

To establish the overall thermal structure of the Martian ionosphere the plan is to observe the electron temperature continuously. The data obtained during the mission will be grouped accordingly season, longitude, latitude, and local time at different heights. The deviation of $T_{e}$ from the mean value which might be found, for instance, during sand storms can thus be isolated and studied in order to establish the relevant processes.

The ionosphere of Mars has never before been studied in a comprehensive and systematic manner, as planned by "NOZOMI". The data obtained by the PET instrument will be a major component of the expected data set, that most likely will advance our understanding of the physical and chemical processes controlling the Martian ionosphere. In this report, we try to have a full description of the PET instrument onboard "NOZOMI", which evolved from rocket and satellite experiments over an extended period of time.

\section{Principle of the Measurement}

When an electrode is immersed in a plasma, characterized by a single electron temperature $T_{e}$, the probe current, expressed in terms of the probe voltage $V$ with respect to the plasma potential in the electron retarding regime, is given as:

$$
I=i_{e} \exp \left(-\frac{e V}{k T_{e}}\right)-i_{i}
$$

with $i_{e}=S_{e} N_{e} \sqrt{\frac{k T_{e}}{2 \pi m_{e}}}$ and $i_{i}=S_{i} N_{i} e V_{s}$.

In the above equation, $S_{e}, N_{e}$ and $T_{e}$ are the effective electron collecting area, electron density and electron temperature, respectively; $k, m_{e}$, and $e$ are Boltzmann's constant, electron mass and electron charge. The ion current, $i_{i}$, is independent of the probe voltage in the case of satellite measurements, because the satellite velocity $V_{s}$ is much higher than the ion thermal velocity. $S_{i}$ is the effective ion collecting area.

When a sine wave of small amplitude ' $a$ ' is superposed onto the sweep voltage, the $V-I$ characteristic shifts as shown in Fig. 1 and the shifted $V-I$ relationship can be written as:

$$
I=i_{e} I_{0}\left(\frac{e a}{k T_{e}}\right) \exp \left(\frac{-e V}{k T_{e}}\right)-i_{i}
$$

where $I_{0}(x)$ is the modified Bessel function of zeroth order with argument $x$. The floating potential at which the probe current is zero can be expressed as

$$
V_{f a 1}=\frac{k T_{e}}{e} \ln \left[\frac{i_{e}}{i_{i}} I_{0}\left(\frac{e a}{k T_{e}}\right)\right] .
$$

Similarly the floating potential without a sinusoidal wave $(a=0)$ is

$$
V_{f}=\frac{k T_{e}}{e} \ln \left(\frac{i_{e}}{i_{i}}\right) .
$$

The shift between the floating potential and the original potential is finally obtained by subtracting Eq. (4) from Eq. (3)

$$
\Delta V_{f a 1}=V_{f a 1}-V_{f}=\frac{k T_{e}}{e} \ln \left[I_{0}\left(\frac{e a}{k T_{e}}\right)\right] .
$$

When a sine wave with amplitude ' $2 a$ ' is applied to the electrode, the floating potential shift is accordingly

$$
\Delta V_{f a 2}=V_{f a 2}-V_{f}=\frac{k T_{e}}{e} \ln \left[I_{0}\left(\frac{2 e a}{k T_{e}}\right)\right] .
$$

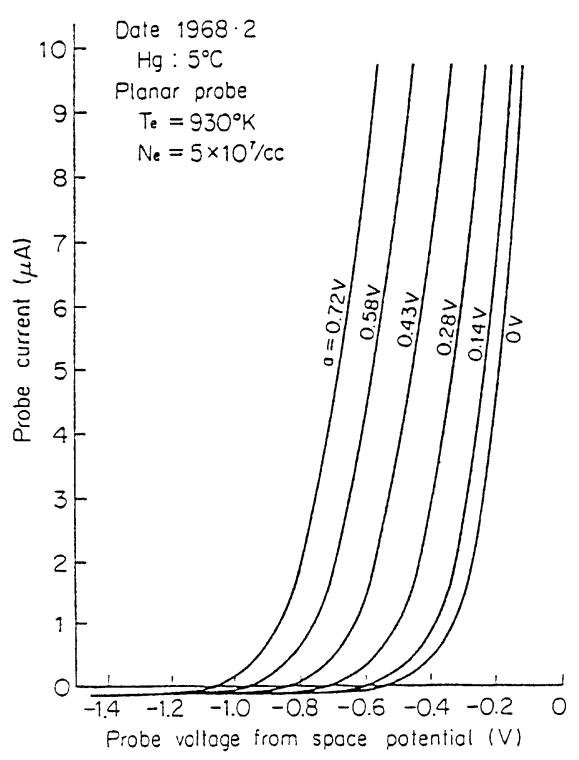

Fig. 1. Principle of the electron temperature probe. The $V-I$ characteristic shifts when sine waves of an amplitude of $0.14,0.28,0.43,0.58$, and $0.72 \mathrm{~V}$ are superposed on the dc probe voltage. $N_{e}$ and $T_{e}$ are $5 \times 10^{7}$ $\mathrm{cm}^{-3}$ and $930 \mathrm{~K}$ respectively. 
The electron temperature is obtained from the individual floating potential shifts when sinusoidal waves of the amplitude $a$ and $2 a$ are successively applied. The first and the second electron temperature estimates $T_{e 1}$ and $T_{e 2}$ are calculated from Eqs. (5) and (6), because $\Delta V_{f a 1}$ and $\Delta V_{f a 2}$ are measured and $k, e$ and $a$ are known. The ratio of the two floating potential shifts which are expressed by Eqs. (5) and (6), can be written as

$$
R=\frac{\Delta V_{f a 1}}{\Delta V_{f a 2}}=\frac{\ln \left[I_{0}\left(\frac{2 e a}{k T_{e}}\right)\right]}{\ln \left[I_{0}\left(\frac{e a}{k T_{e}}\right)\right]} .
$$

Equation (7) provides a third electron temperature estimate $T_{e 3}$. When the electrons in the plasma are Maxwellian, the three electron temperatures $T_{e 1}, T_{e 2}$, and $T_{e 3}$ should be the same. If all these electron temperatures differ from one another, it can be inferred that the electrons in the plasma exhibit either a high energy tail or that populations with different temperatures are present. The effects of the coexistence of electron populations with different temperatures on the measured $T_{e}$ will be discussed later in this paper.

The basic instrument circuit characterizing the measurement principle is shown in Fig. 2. The sine waves of amplitudes ' $a$ ' and ' $2 a$ ', and of frequency, $f$ are fed to an electrode through a capacitor $C_{1}$. The impedance of the feeding capacitance, $\frac{1}{2 \pi f C_{1}}$ should be low enough to neglect the impedance of the sheath formed around the electrode as we will discuss later. The signal at the electrode is also shown in this figure where potential shifts due to the nonlinearity of the sheath resistance are superposed on the original sine wave (dashed line, lower left). After we remove the sine wave component by a simple $C_{2} / R_{2}$ filter, the floating potential shifts purely generated by the sine wave remains as shown in the upper right of Fig. 2.

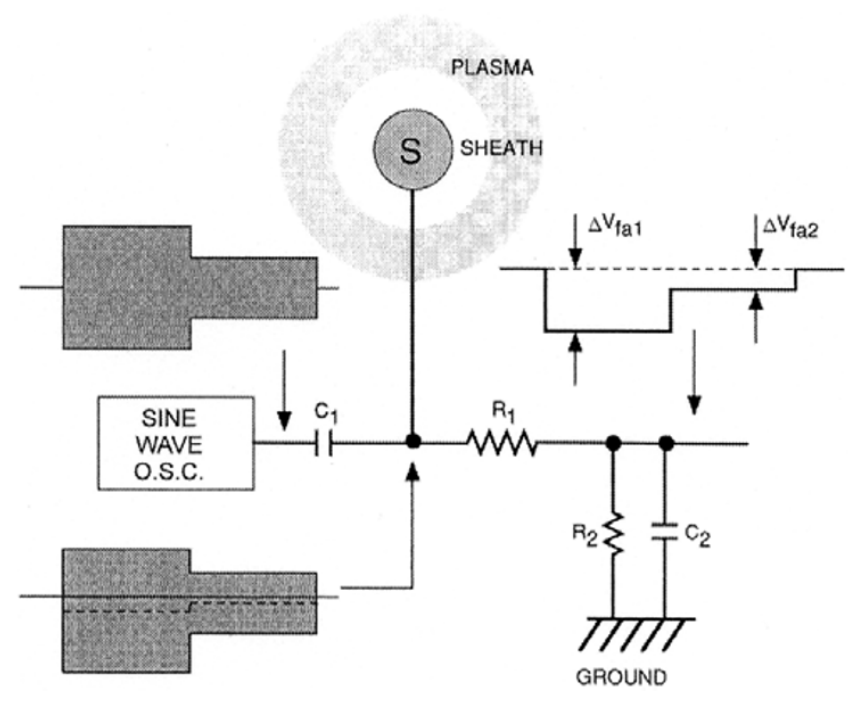

Fig. 2. Basic electronic circuit of the electron temperature probe. The sine wave is applied to the electrode through the capacitor $C_{1}$. It is thereby superposed on the floating potential shift generated by the application of this wave. The signal is then filtered by a $C_{2} R_{2}$ circuit yielding the pure floating potential. The sine wave is modulated at three different amplitudes $(500 \mathrm{mV}, 250 \mathrm{mV}$ and $0 \mathrm{~V}$, shown in gray).
For the actual circuit, a circular electrode is split into two parts with a spacing in-between (two semicircular plates). A sine wave is applied to one of the divided electrodes, whilst, no signal is applied to other divided electrode. The signal at the former plate, $V_{f 1}$ is

$$
V_{f 1}=V_{f}+\Delta V_{f a 1}\left(\Delta V_{f a 2}\right)
$$

where $V_{f}$ is the floating potential without signal. The signal at the second half electrode, $V_{f 2}$ is;

$$
V_{f 2}=V_{f}
$$

If we feed the above two signals through a resistor $R_{1}$ to a differential amplifier of gain $G$ with an input resistor of $R_{2}$ as shown in Fig. 2, we finally obtain the output;

$$
\begin{aligned}
\Delta V_{f} & =G\left(V_{f 1}-V_{f 2}\right) \frac{R_{2}}{R_{1}+R_{2}} \\
& =G \Delta V_{f a 1}\left(\Delta V_{f a 2}\right) \frac{R_{2}}{R_{1}+R_{2}} .
\end{aligned}
$$

Therefore pure floating potential shifts generated by the application of a sine wave to the electrode can be detected with a differential amplifier and noise components as well as the original floating potential is eliminated. Strictly speaking, however, the floating potentials of each plate differ slightly due to a nonuniform work function of the electrode surfaces and this cannot be removed at the amplifier output, but this voltage remains within the allowed voltage dynamic range.

\section{Examination of the Probe Characteristics 3.1 Frequency of the sine wave}

The electron temperature determination described above includes several factors that need to be taken into account during the design phase. The first factor is related to the frequency of the sine wave. This frequency should not be lower than the ion plasma frequency, because a small floating potential shift would result from the nonlinearity of the ion current response. The ion plasma frequency is about 2-3 $\mathrm{kHz}$ in a plasma with a density of $10^{5} \mathrm{~cm}^{-3}$ and the driving frequency is normally chosen close to $30 \mathrm{kHz}$ which is much higher than the normal electron plasma frequency.

\subsection{Effect of sheath impedance}

The other factor relates to the loading of the sine wave applied to the active plate. It has to be taken into account when the dynamic impedance of the plasma is low, and can be easily quantified. The probe circuit immersed in a plasma is equivalently expressed as shown in Fig. 3. The dependence of the electron current to the probe on the applied voltage near the floating potential can be derived from Eqs. (1) or (4). The dynamic impedance at the floating potential is thus given by

$$
\left(\frac{d V}{d I}\right)_{a t V_{f}}=Z_{d}=\frac{k T_{e}}{e i_{i}} .
$$

The area $A$ of the plate $(1 / 2$ of a $150 \mathrm{~cm}$ diameter circular disk) is approximately $88.3 \mathrm{~cm}^{2}$ so that $i_{i}$ can be expressed as

$$
i_{i}=A N_{i} V_{s} \times 1.6 \times 10^{-19} \cos \theta \quad(\text { in } A)
$$




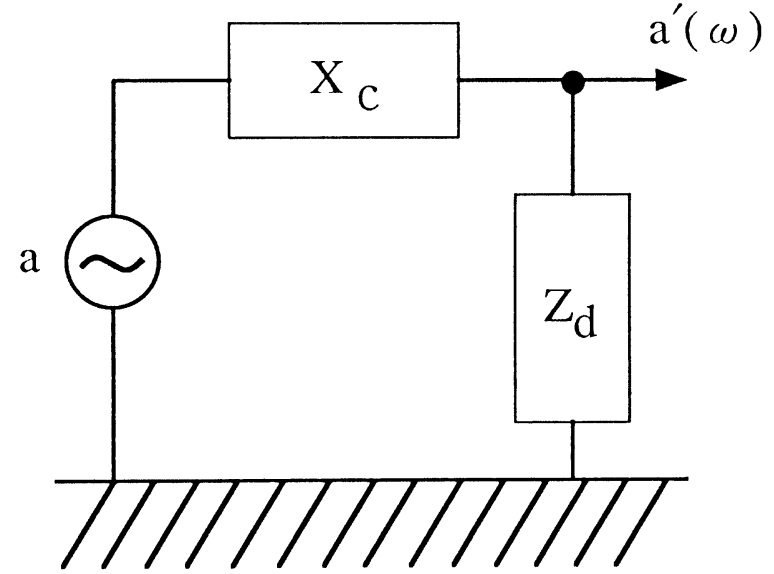

Fig. 3. Equivalent circuit of the floating potential detection. $X_{c}$ is the reactance of the capacitive coupling of the wave oscillator a and the sensor plate, $Z_{d}$ is the dynamic impedance of the plasma.

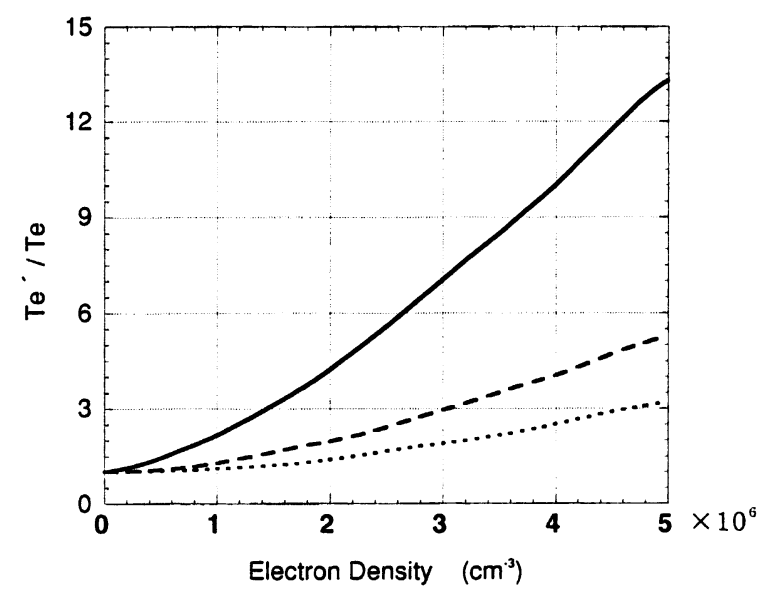

Fig. 4. Effect of the reduced amplitude caused by the feeding capacitor Plotted is the ratio of apparent to true electron temperature as a function of electron density for three different capacitors $C_{1}$ (solid line is for 1000 $\mathrm{pF}$, dashed line for $2000 \mathrm{pF}$, and dotted line for $3000 \mathrm{pF}$ ) for zero attack angle. Amplitude of the signal is $0.5 \mathrm{~V}$. The true temperature is $500 \mathrm{~K}$.

here $\theta$ is the angle between the probe normal and the satellite motion (called angle of attack in the following). For a satellite velocity $V_{s} \approx 7.5 \mathrm{~km} \mathrm{sec}^{-1}$, this leads to an expression for $Z_{d}$, which is assumed here to be resistive.

$$
Z_{d} \approx 0.81 \times 10^{7} \frac{T_{e}}{N_{e}} \cos \theta \quad(\text { in } \Omega)
$$

where $N_{e}\left(=N_{i}\right)$ is expressed in $\mathrm{cm}^{-3}$.

The reactance of the capacitive coupling to the plate from the driving oscillator is $X_{c}=\frac{1}{2 \pi f C_{1}}$ so that the actual voltage amplitude at the plate is given by

$$
a^{\prime}=a \sqrt{\frac{Z_{d}^{2}}{Z_{d}^{2}+X_{c}^{2}}} .
$$

Equation (14) shows that the amplitude which is actually applied to the electrode through a driving oscillator is reduced if the driving capacity $C_{1}$ is not sufficiently large to neglect the sheath impedance.

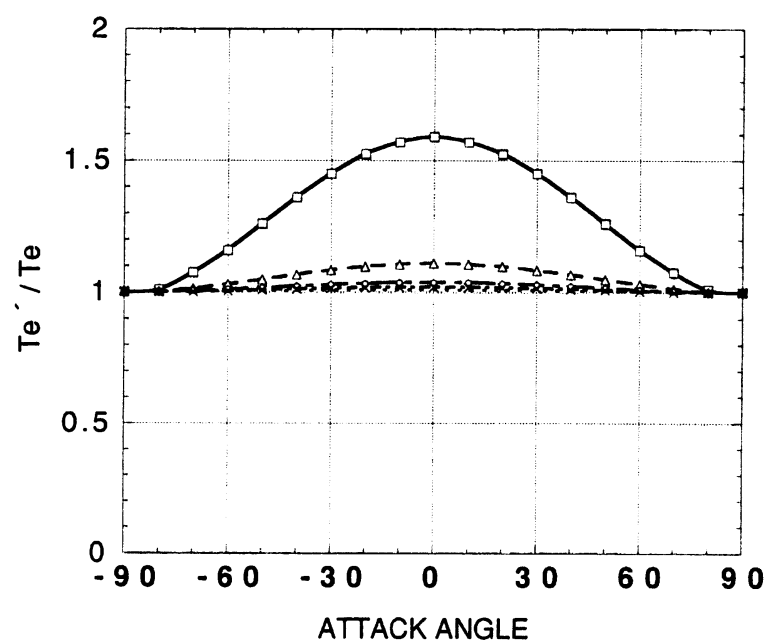

Fig. 5(a). Effect of the angle of attack $\theta$ on the electron temperature evaluation for a feeding capacitance of $1000 \mathrm{pF}$ and four different electron temperatures $(\square: 500 \mathrm{~K}, \triangle: 1000 \mathrm{~K}, \bigcirc: 1500 \mathrm{~K}, \times: 2000 \mathrm{~K})$. A plasma with a density of $10^{6} \mathrm{~cm}^{-3}$ was assumed, and $T_{e}$ was calculated from the floating potential shift caused by a sine wave of $0.25 \mathrm{~V}$ amplitude.

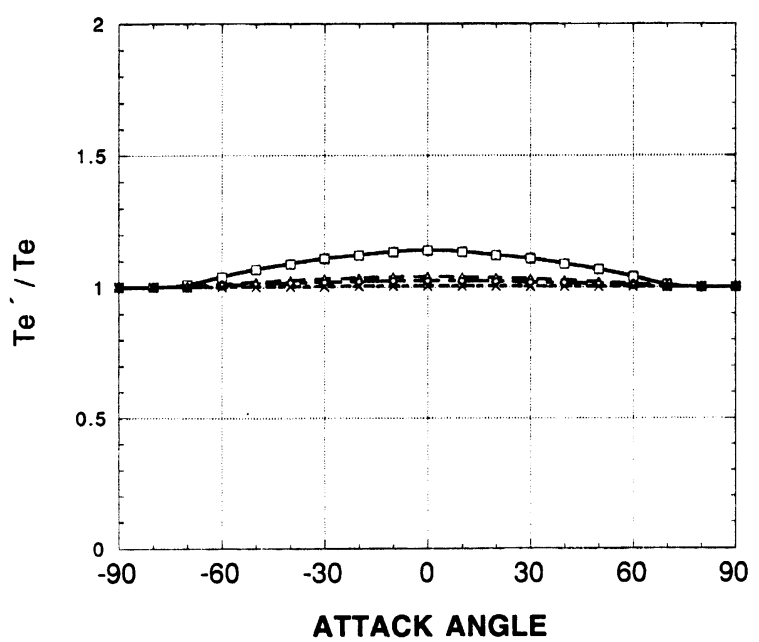

Fig. 5(b). The same as for Fig. 5(a), but $T_{e}$ was calculated from the ratio of the two floating potential shifts (Eq. (7)).

Figure 4 shows the electron temperature, $T_{e}^{\prime}$ erroneously calculated by using the reduced sinusoidal signal, as a function of electron density for three feeding capacitors $(1000$, 2000 , and $3000 \mathrm{pF}$ ), and for $\theta=0 . T_{e}$ is fixed at $500 \mathrm{~K}$ which should be valid at a height of about $200 \mathrm{~km}$ in the Mars ionosphere according to the Viking measurements.

It is obvious that the electron temperature is considerably overestimated at high ambient electron densities when the feeding capacitor is too small. It should be noted however that the maximum electron density in the Mars ionosphere is around $5 \times 10^{5} \mathrm{~cm}^{-3}$. Therefore the electron temperature error is less than $5 \%$ if a capacity of $C_{1}=2000 \mathrm{pF}$ is used.

The influence of the angle of attack on the electron temperature calculated from the floating potential shift for a sine wave of $0.25 \mathrm{~V}$ amplitude (Fig. 5(a)) and from the ratio of the two floating potential shifts (Fig. 5(b)) is also examined 
(for a constant feeding capacitance of $C_{1}=1000 \mathrm{pF}$ ). The electron temperature is varied from $500 \mathrm{~K}$ to $2500 \mathrm{~K}$ assuming a constant plasma density of $10^{6} \mathrm{~cm}^{-3}$. At zero angle of attack the calculated electron temperature is 1.6 times higher than the true $T_{e}=500 \mathrm{~K}$, but the error decreases with the ambient electron temperature: the ratio $T_{e} / T_{e}^{\prime}$ becomes 1.1 and 1.005 for $T_{e}=1000 \mathrm{~K}$ and $2500 \mathrm{~K}$, respectively. This indicates that electron temperatures higher than $1500 \mathrm{~K}$ can be correctly measured for $C_{1}=1000 \mathrm{pF}$ and $N_{e}=10^{6}$ $\mathrm{cm}^{-3}$.

Figure 5(b) shows that the effect of the reduced amplitude of the sine wave is smaller if we calculate the electron temperature from the ratio of the two floating potential shifts as in the case of nonnegligible $Z_{d}$. The calculation was carried out with the same parameters $\left(C_{1}=1000 \mathrm{pF}\right.$, and $N_{e}=10^{6} \mathrm{~cm}^{-3}$ ) and for the same five electron temperatures as in Fig. 5(a). In Fig. 5(b), a true $T_{e}=500 \mathrm{~K}$ yields $575 \mathrm{~K}$ at zero angle of attack, while from Fig. 5(a), $T_{e}=800 \mathrm{~K}$ follows.

\subsection{Effect of the input resistance on the amplifier output}

The floating potential shifts expressed by Eq. (10) appears at the output of the amplifier when the detecting resistance $\left(R_{1}+R_{2}\right)$ is much higher than $Z_{d}$. When $Z_{d}$ is high, the resistance $\left(R_{1}+R_{2}\right)$ reduces the output amplitude of the floating potential shifts $\Delta V_{f}$ to

$$
\Delta V_{\text {fred }}=\frac{G R_{1} \Delta V_{f}}{Z_{d}+R_{1}+R_{2}}
$$

where resistances $R_{1}+R_{2}$ are the same as indicated in Fig. 1 and $Z_{d}$ is expressed by Eq. (13). Suppose that $N_{e}=500$ $\mathrm{cm}^{-3}, T_{e}=5000 \mathrm{~K}$, and $\theta=0, Z_{d}=81 \mathrm{M} \Omega, \Delta V_{\text {fred }}$ is reduced tremendously. The electron temperature which is calculated from the reduced $\Delta V_{\text {fred }}$ is higher than the true value. The effect of the input resistance on the electron temperature calculated is removed if we take the ratio of the output floating potential shifts of the amplifier.

\subsection{Distortion of the output signal}

The analysis described above shows that the capacity $C_{1}$ should be as large as possible. There is however another effect that limits this value, namely its influence on the shape of the output signal.

In order to visualize this effect, the output signal of the electron temperature probe is monitored by an oscilloscope for feeding capacities between $1000 \mathrm{pF}$ and $6000 \mathrm{pF}$ (Fig. 6). It can be seen that for $6000 \mathrm{pF}$ the detected signal does not reach the final level due to the large time constant $C_{1} Z_{d}$ (c.f. Fig. 2). As the capacitance is reduced from $6000 \mathrm{pF}$ to 1000 $\mathrm{pF}$, the output signal starts to represent the ideal wave shape. It should be noted, however, that the amplitude of the output signal decreases as the capacitance decreases from $6000 \mathrm{pF}$ to $1000 \mathrm{pF}$ according to the Eq. (14).

As a compromise between the two requirements, a capacitor of $C_{1}=2000 \mathrm{pF}$ was selected for the flight instrument.

\subsection{Signal quality for the electron temperature evalua-} tion

The quality of the sinusoidal signal which is applied to the electrode influences the accuracy of the $T_{e}$ measurements. In order to evaluate the effect of a distorted sine wave on the accuracy of the measurement, a voltage

$$
\tilde{a}(\omega)=a(\sin \omega t+k \sin n \omega t)
$$
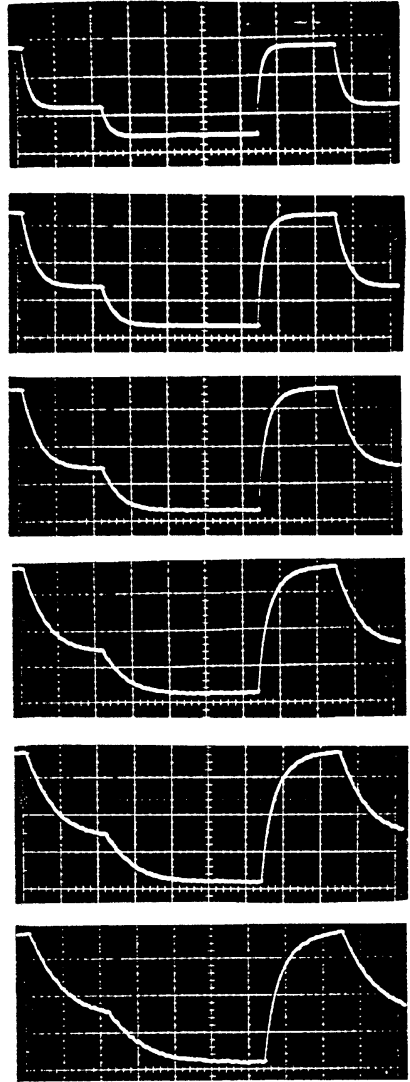

Fig. 6. Effect of the time constant $C_{1} Z_{d}$ on the shape of the output signal for 6 different values of $C_{1}$. The time needed to reach the final level increases with increasing capacitance. The different levels of the output signal in the six panels is a consequence of the sinusoidal signal amplitude, raising as the capacitance increases from the top to the bottom, $1000 \mathrm{pF}, 2000$ $\mathrm{pF}, 3000 \mathrm{pF}, 4000 \mathrm{pF}, 5000 \mathrm{pF}$ and $6000 \mathrm{pF}$. The amplitude of the output increases with increasing feeding capacitance, because the amplitude of the sinusoidal signal at the end of the electrode is adjusted for the feeding capacitance of $1000 \mathrm{pF}$. Vertical axis; $0.5 \mathrm{~V} /$ div., horizontal axis; $0.1 \mathrm{sec} /$ div.

which includes higher harmonic components is applied to the electrode and the electron temperature is calculated from the floating potential which is obtained by averaging the distorted current. The results are shown in Figs. 7(a) and 7(b), for three different electron temperatures as a function of $k$ and $n=2$ and 3 , respectively. Figure 7 indicates that the electron temperature is less influenced by the distortion of the wave with an even number of $n: T_{e}=500 \mathrm{~K}$ and $k=5 \%$ produces a $\widetilde{T}_{e}$ which is 0.99 times lower than the true value, while $\widetilde{T}_{e}$ becomes $22.5 \%$ higher than the true value for $n=3$ (Fig. 7(b)). This calculation concludes that $T_{e}$ can be accurately obtained if the distortion of the signal applied to the electrode is less than $1 \%$, a level which can be easily achieved without any serious technical difficulty.

\subsection{Effects of two electron temperatures}

In order to get a rough idea of the reaction of the probe to a plasma with two electron populations with different temperatures, we performed the following calculations. A plasma with five different electron temperatures $\left(T_{e}=500,1000\right.$, 1500,2000 , and $2500 \mathrm{~K}$ ) was assumed to contain a second population with a different temperature and a certain mixing ratio. For this mixture the apparent electron temperature 


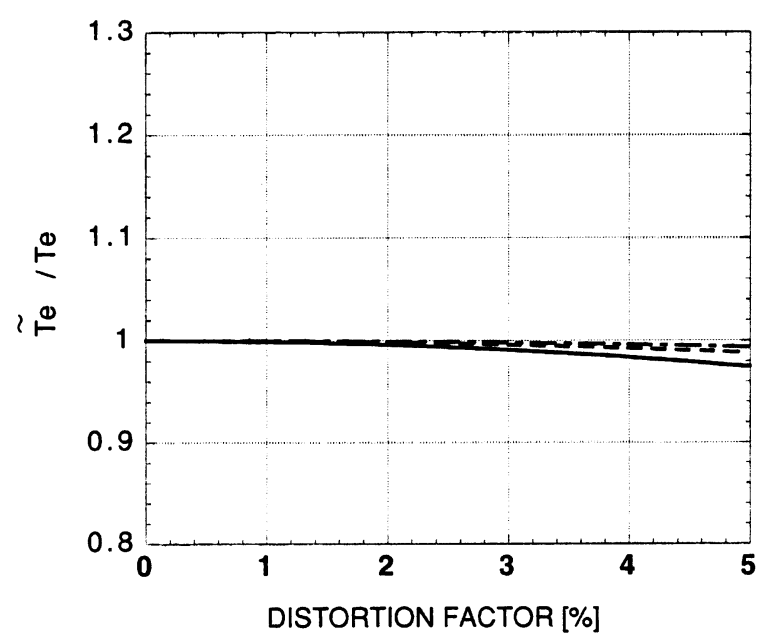

Fig. 7(a). Effect of the presence of higher harmonics in the applied sine wave on the electron temperature. The ratio of apparent to true $T_{e}$ (full line: $500 \mathrm{~K}$, dashed line: $1000 \mathrm{~K}$, dot-dashed line: $2000 \mathrm{~K}$ ) is plotted as a function of the distortion factor $k$. A sine wave amplitude of $0.5 \mathrm{~V}$ and a mode number $n=2$ was assumed.

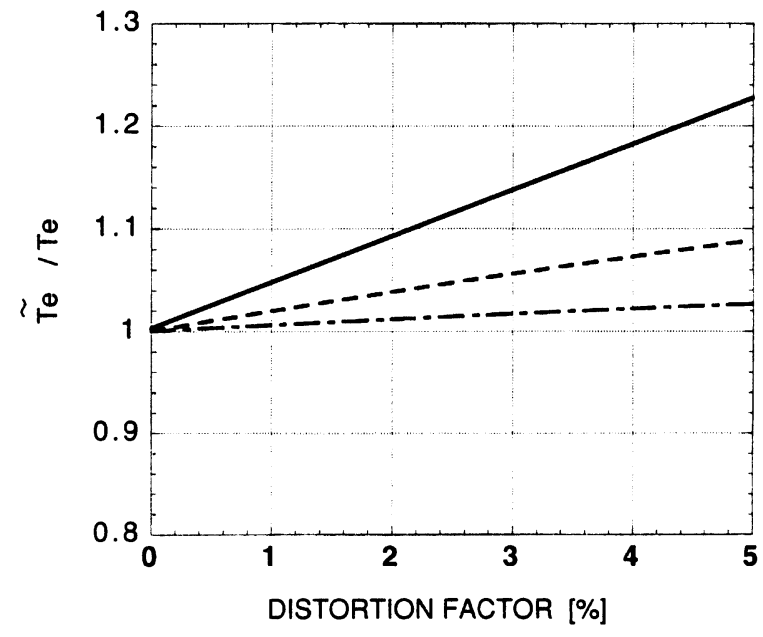

Fig. 7(b). The same as for Fig. 7(a) but for a mode number $n=3$.

$T_{e}^{*}$ obtained from the floating potential shifts was calculated. Figure 8 shows the result for a mixing ratio of $0.1 \%$ and the second temperatures indicated on the abscissa. A sine wave amplitude of $0.5 \mathrm{~V}$ was assumed for the calculations. The figure shows for instance, that for a plasma of $2000 \mathrm{~K}$ containing $0.1 \%$ of electrons with $3000 \mathrm{~K}$ ( or $4000 \mathrm{~K}$ ), the probe would return $T_{e}=2050 \mathrm{~K}$ (or $2600 \mathrm{~K}$ ), respectively. Enhancing the mixing ratio to $1 \%$ the probe would give $T_{e}=2600 \mathrm{~K}$ (or more than $10000 \mathrm{~K}$ ) for the same temperature combinations. The conclusion from these calculations is that the presence of only $1 \%$ of suprathermal electrons influences the accurate measurement of $T_{e}$ drastically.

\section{The Electron Temperature Probe Onboard "NOZOMI"}

A glass fiber board $1.6 \mathrm{~mm}$ thick was used to make a circular sensor plate of $150 \mathrm{~mm}$ diameter. The circular plate is split into two semicircular disks with a gap of $10 \mathrm{~mm}$ in-

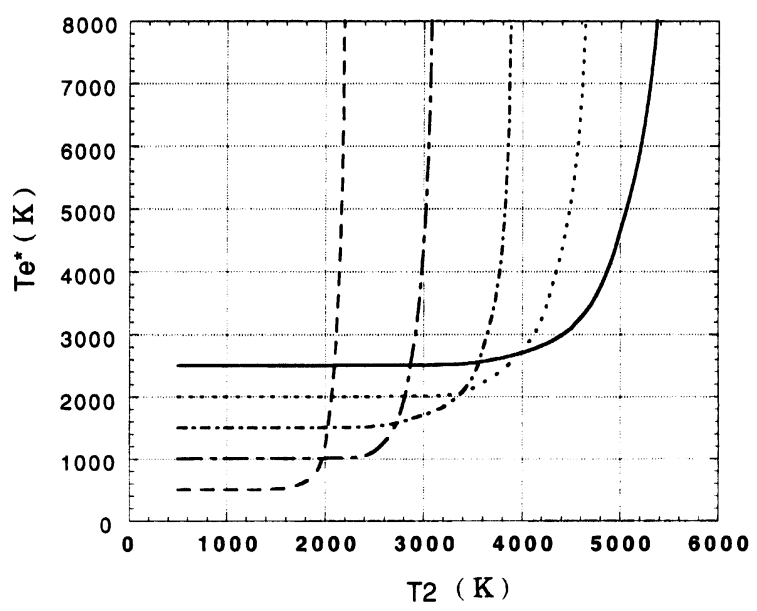

Fig. 8. Effect of two plasma populations with different temperatures on the $T_{e}$ value returned by the probe. The curves are calculated for a plasma with five different temperatures $(500 \mathrm{~K}, 1000 \mathrm{~K}, 1500 \mathrm{~K}, 2000 \mathrm{~K}, 2500 \mathrm{~K}$ from the left to the right) containing a second electron population with a mixing ratio of $0.1 \%$ and the temperatures indicated on the abscissa. A sine wave amplitude is $0.5 \mathrm{~V}$.

between. A sinusoidal RF signal is applied to one of the semicircular plates (Signal Electrode in Fig. 9). No signal is applied to other plate (Reference Electrode). The weight is $150 \mathrm{~g}$. Two sensor electrodes (S1 and S2) are placed at the end of two opposite solar cell panels which are located vertical to the satellite spin axis as shown in Fig. 10. The normal of the sensor electrodes are fixed parallel to the solar cell panels in order to avoid the exposure to the sun light which produces secondary electrons from the electrode surface. The fundamental structure of the system is the same as the one used on all earth orbiting satellites in the past, with some minor modifications. A Colpitz type oscillator with an original frequency of $7.168 \mathrm{MHz}$ is used, and the original frequency is converted to $28 \mathrm{kHz}$. This value was chosen in order to minimize a possible interference with the wave receivers onboard "NOZOMI". In the real Mars ionosphere, as the signal from the sensor electrode leaks to the plasma environment through the sheath, interference may arise from the electrostatic coupling between the sensor electrode and the VLF receiver. However the interference can be removed, if necessary, as the frequency of the signal is known.

The amplitudes of the sine wave applied to the electrode, $a$ and $2 a$ are adjusted to $250 \mathrm{mV}$ and $500 \mathrm{mV}$. The variation of the amplitude is within $1 \%$ over a temperature range from $-40 \mathrm{~K}$ to $+50 \mathrm{~K}$. The distortion factor of the sine wave is less than $1 \%$.

The over all gain of the differential amplifier including the buffer amplifier is 6.22 for both electronics. The floating potential of the reference electrode with respect to the satellite frame is measured with a buffer amplifier with a gain of 0.91 .

The electronics of the PET are accommodated in one box together with those of the magnetometer, and a UV imager, in order to minimize the total weight of the payload. Electric power for the PET will be supplied through the magnetometer and the power is switched on/off simultaneously with the magnetometer. Power consumption is $16 \mathrm{~mA}$ at $+/-12 \mathrm{~V}$ and $3 \mathrm{~mA}$ at $5 \mathrm{~V}$. The lower limit of the ambient electron 


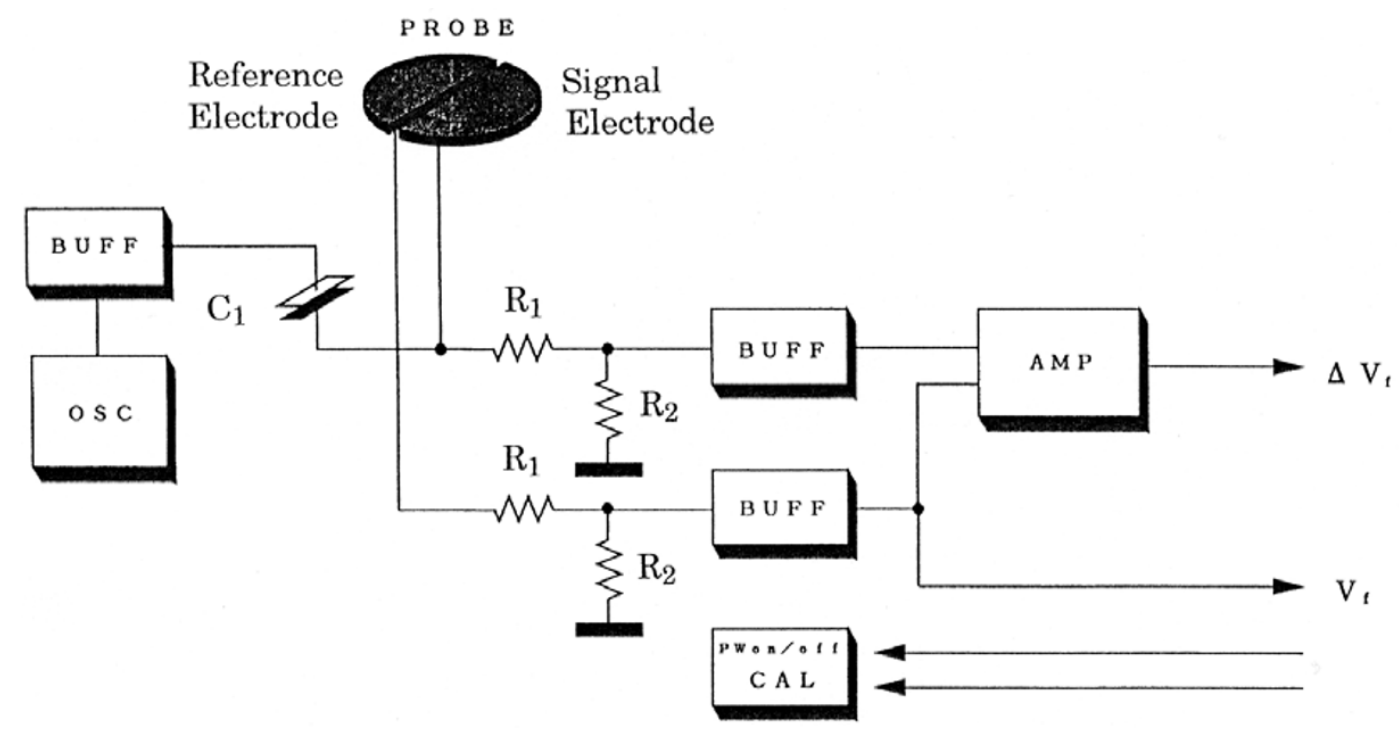

Fig. 9. Block circuit diagram of the PET instrument.

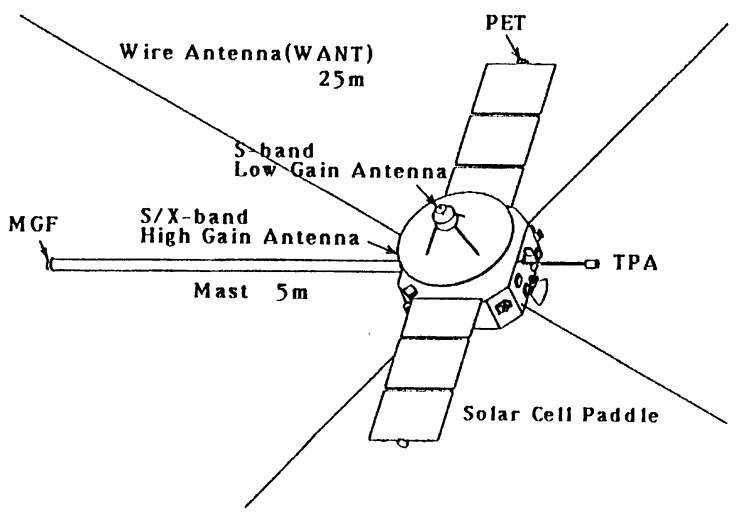

Fig. 10. Location of the PET sensors on the "NOZOMI" spacecraft.

density for a reliable measurement of $T_{e}$ is $10^{3} \mathrm{~cm}^{-3}$. Accordingly, night time measurements in the Mars ionosphere may be limited to the height range of the density maximum $(150-200 \mathrm{~km})$. When the electron density is below $10^{3} \mathrm{~cm}^{-3}$ (see Fig. 11), no measurements will be possible.

The instruments, based on the same measurement principle as described above, have been used for the sounding rocket in Japan, as well as in other countries, and the instruments were also onboard five Japanese scientific satellites. The results obtained from these long term experiments confirmed the high reliability and stable performance (Oyama, 1991).

\section{Data Acquisition}

The spacecraft "NOZOMI" usually rotates once per $8 \mathrm{sec}-$ onds along the satellite mechanical axis. In Fig. 12(a), a time chart of the sine wave for the two sensor electrodes is shown. All data samplings related to the electron temperature are normally referred to the sun direction. The figure shows an index pulse (called sun pulse in the following), which appears once per spin, a series of 256 sampling pulses, and the sinusoidal signal which are applied to the sensor S1 and S2.

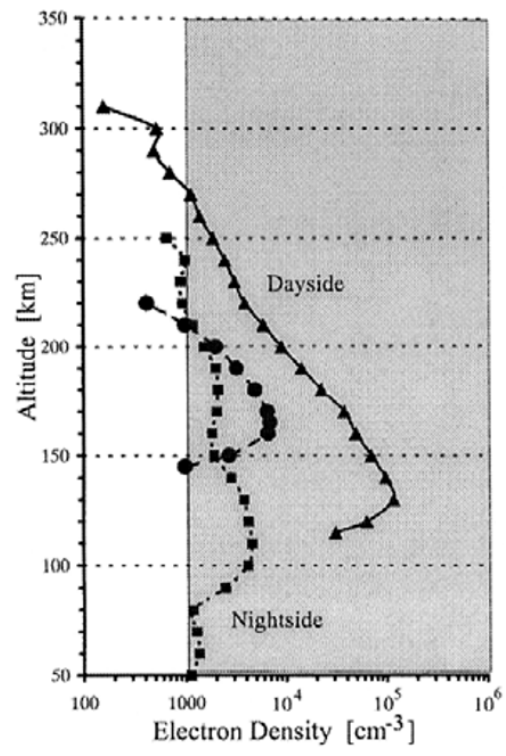

Fig. 11. Height ranges where the electron temperature probe can give a reliable data (grey zone). Three typical electron number density profiles are shown: $\boldsymbol{\Delta}$ from retarding potential trap on board Viking II (Hanson et al., 1988), $\mathbf{\square}$ from radio occultation technique (Verigin et al., 1991) and, from theory for the night (Verigin et al., 1991).

The sinusoidal signal of amplitude, $a=0.5 \mathrm{~V}$, is applied to the sensor S1, $62.5 \mathrm{~ms}$ before the rising phase of the first sun pulse. At $62.5 \mathrm{~ms}$ after the sun pulse it is reduced to $a=0.25 \mathrm{~V}$, and $187.5 \mathrm{~ms}$ after the sun pulse it becomes zero. The zero level stays for $250 \mathrm{~ms}$, then the second sequence starts. The sinusoidal signal for the sensor S2 starts exactly at the rising phase of the sun pulse, but has otherwise the same timing as $\mathrm{S} 1$. This sequence is repeated 16 times during one spin period ( 8 seconds).

The output signals from which the electron temperature is calculated consist of the three levels of the floating potential shifts according to the application of the sine waves with 


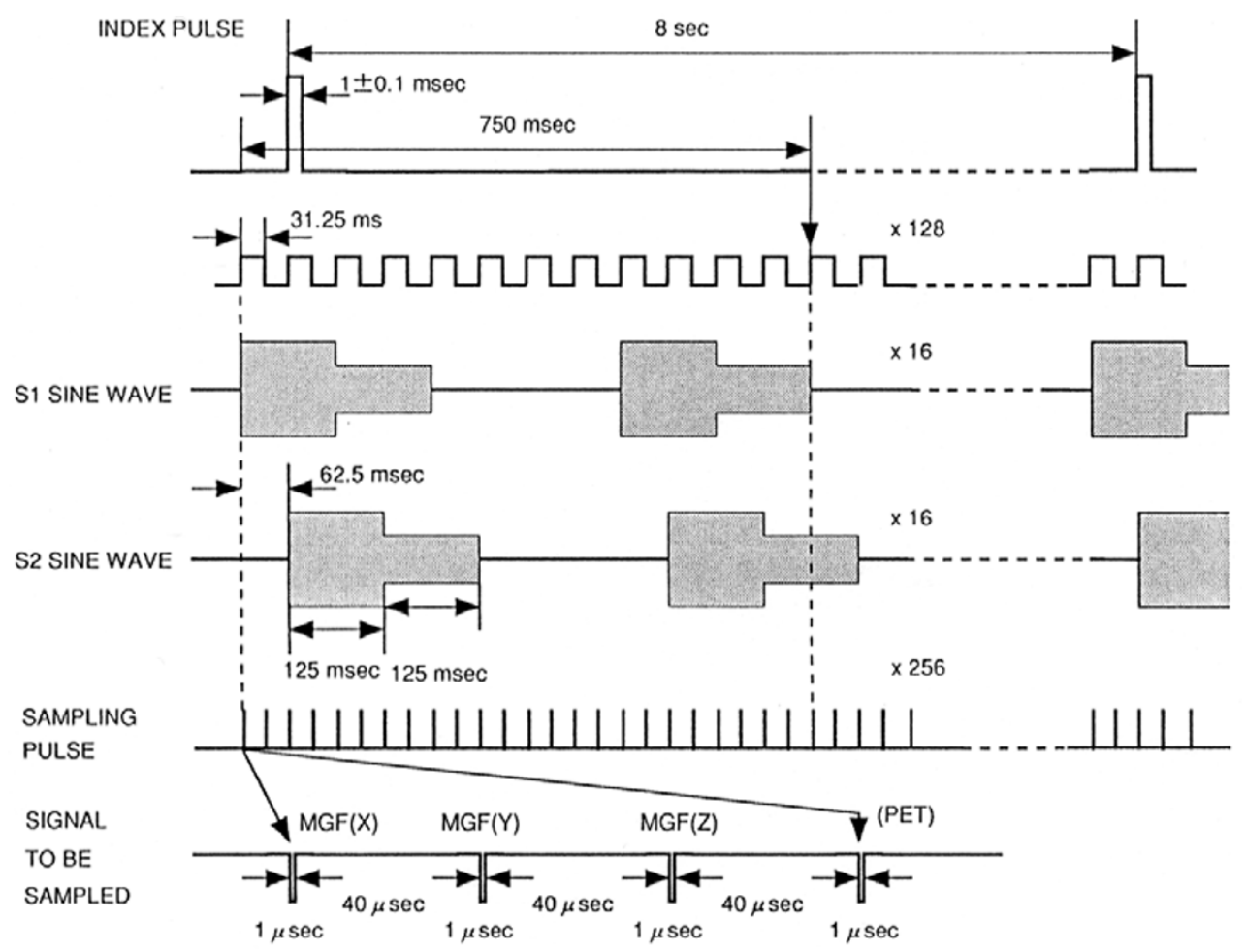

Fig. 12(a). Time sequence for the data sampling with reference to the index pulse (sun pulse). The gray shaded areas indicate the time intervals when the sine wave with amplitude $a$ or $2 a$ is applied to the sensor.

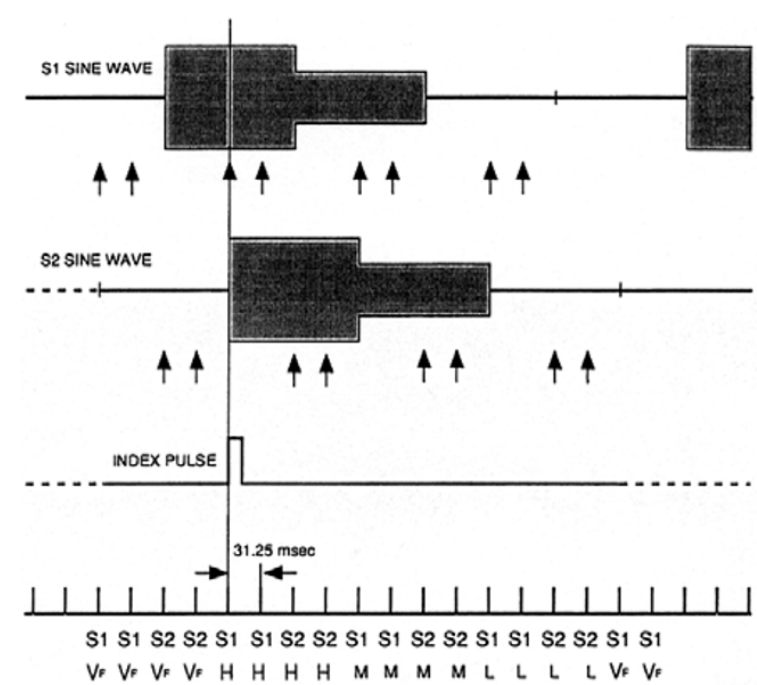

Fig. 12(b). Expanded part of the time sequence. The PET output signal is sampled at the times indicated by the arrows.

$a=0.5,0.25$ and $0 \mathrm{~V}$ to the sensor electrode, and of the floating potential of the reference electrode with respect to the spacecraft frame. The set of these four signals are named $H, M, L$, and $V_{f}$ level. These signal levels are sampled twice with a $31.25 \mathrm{~ms}$ gap during the last half period $(62.5 \mathrm{~ms})$ of the signal application as shown in Fig. 12(b). The output from the instruments are fed to an analog/digital converter, which digitizes the levels with 16 bits for $\pm 4.5 \mathrm{~V}$ at a $64 \mathrm{k}-$ bit data rate. The digital signal for the PET amounts to 256 words (16 times per spin $\times 2$ samples at 4 different levels,

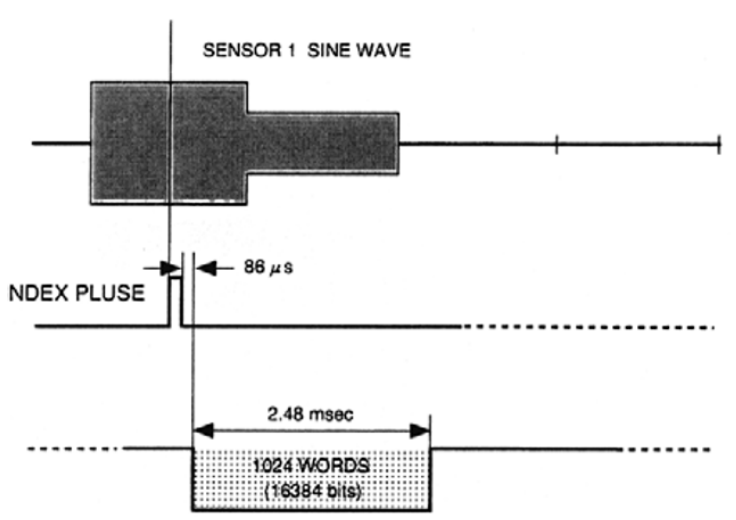

Fig. 13. Timing of the electron temperature data storing.

for 2 sets of the instruments). Out of the 16 bits, the MSB is used as a sign bit, and the LSB for monitoring of the high frequency pulse of the top side sounder. As the PET can give meaningful data only in the plasma environment with an electron density above $1000 \mathrm{~cm}^{-3}$, the data have to be sampled in a periapsis mode (telemetry format-16, which is operated at a rate of $64 \mathrm{k}-16 \mathrm{k}$ bits $/ \mathrm{sec}$ ).

The total sampling time for one set of $T_{e}$ data is $0.5 \mathrm{sec}-$ ond. This means that $16 T_{e}$ data sets are obtained during one satellite spin with an angular resolution of 20 degrees for each sensor. When the telemetry bit rate is $512 \mathrm{bps}$ (Format-17), $T_{e}$ is only sampled every 64 seconds. In this situation, if it happens, only the performance of the instrument is monitored. Command signals to control the instruments are; POWER 
ON/OFF, CAL and SWEEP OFF. During the CAL mode, dc signals with an amplitude ratio of 2 are applied to check the amplifier gain, but the sinusoidal signal is still applied to the probe. The command SWEEP OFF, which was prepared to avoid a possible interference with the VLF receiver disconnects the output signal from the original oscillator.

\section{Storing of the Sampled Data}

One data unit (1024 words) from the magnetic field instrument ( 768 words) and PET (256 words) which is stored between two sun pulses is read out $86 \mu$ s after the second sun pulse during $2.48 \mathrm{~ms}$ as illustrated in Fig. 13. The first signal in the data unit, the floating potential shift of sensor 1 caused by $a=0.5 \mathrm{~V}$ is sampled at $31.25 \mathrm{~ms}$ after the first pulse, and the last signal in the data unit ends with the floating potential shift of sensor 1 caused by $a=0.5 \mathrm{~V}$, sampled at the rising edge of the second sun pulse (see also Fig. 12(b)).

Acknowledgments. We would like to express our sincere gratitude to the rocket launching staff of ISAS for their cooperation. The electronics of the electron temperature probe was designed by H. Takano of Meiwa System Co. 1td. and manufactured by Meisei Electric Company. Acknowledged are also all personnel and institutions who assured the successful launch.

\section{References}

Choi, Y. W., J. Kim, K. W. Min, A. F. Nagy, and K.-I. Oyama, Effect of the magnetic field on the energetics of Mars ionosphere, Geophys. Res. Lett.,
25, 2753-2756, 1998.

Cole, K., Origin of flux ropes in the Venus ionosphere, J. Geophys. Res., 99 , 14951-14958, 1994.

Cravens, T. E., T. I. Gombosi, J. Kozyra, A. F. Nagy, L. H. Brace, and W. C. Knudsen, Model calculations of the dayside ionosphere of Venus: Energetics, J. Geophys. Res., 85, 7778-7786, 1980.

Hanson, W. B. and G. P. Mantas, Viking electron temperature measurements; evidence for a magnetic field in the martian ionosphere, J. Geophys. Res., 93, 7538-7544, 1988.

Hanson, W. B., S. Sanatani, and D. R. Zuccaro, The martian ionosphere as observed by the Viking retarding potential analyzers, J. Geophys. Res., 82, 4351-4363, 1988.

Hoegy, W. R., L. H. Brace, and W. T. Kasprzak, Small scale plasma, magnetic, and neutral density fluctuations in the nightside Venus ionosphere, J. Geophys. Res., 95, 4085-4102, 1990.

Oyama, K.-I., Electron temperature measurements carried out by Japanese scientific satellites, Adv. Space Res., 11(10), 109-158, 1991.

Verigin, M. I., K. I. Gringauz, N. M. Shutte, S. A. Haider, K. Szego, P. Kiraly, A. F. Nagy, and T. I. Gombosi, On the possible source of the ionization in the nighttime martian ionosphere 1. Phobos 2 HARP electron spectrometer measurements, J. Geophys. Res., 96, 19307-19313, 1991.

Zhang, M. H. G., J. G. Luhmann, A. J. Kliore, and J. Kim, A post-pioneer Venus reassement of the Martian dayside ionosphere as observed by radio occultation methods, J. Geophys. Res., 95, 14829-14839, 1990a.

Zhang, M. H. G., J. G. Luhmann, and A. J. Kliore, An observational study of the nightside ionospheres of Mars and Venus with radio occultation methods, J. Geophys. Res., 95, 17095-17102, 1990b.

K. Oyama (e-mail: oyama@bochan.ted.isas.ac.jp), T. Abe (e-mail: abe@stp.isas.ac.jp), K. Schlegel (e-mail: schlegel@linmpi.mpg.de), A. Nagy (e-mail: anagy@umich.edu), J. Kim (e-mail: jkim@viva.kari.re.kr), and K. Marubashi (e-mail: kmaru@crl.go.jp) 